\title{
Hidden strange pentaquark states in constituent quark models
}

\author{
Xuejie Liu, Hongxia Huang, ${ }^{*}$ and Jialun Ping ${ }^{\dagger}$ \\ Department of Physics, Nanjing Normal University, Nanjing, Jiangsu 210097, China
}

(Received 19 August 2018; published 26 November 2018)

\begin{abstract}
In the framework of the chiral quark model, we investigate the hidden strange pentaquark system of the $N \phi$ state with quantum numbers of $I J^{P}=\frac{1}{2}^{-} \frac{3}{2}^{-}$. The results show that the $N \phi$ state can be bound through the interaction of the $\sigma$ meson exchange plus the effect of channel coupling, which means that the effect of channel coupling has an influence on the existence of this bound state.
\end{abstract}

DOI: 10.1103/PhysRevC.98.055203

\section{INTRODUCTION}

Quantum chromodynamics (QCD) is the underlying theory of the strong interaction. However, it is difficult to study structures of hadrons and hadron-hadron interactions directly because of the nonperturbative properties of QCD in the low energy region, although lattice QCD has made impressive progress on nucleon-nucleon interactions and multiquark systems [1-3]. The QCD-inspired quark models are still the main approach to study hadron-hadron interactions and multiquark states. QCD does not forbid the existence of exotic hadronic states other than $q q q$ baryons and $q \bar{q}$ mesons, such as glueballs (without quark/antiquark), hybrids, multiquark states, and hadron molecules. Searching for all these kinds of states has a long history, and various methods have been applied, such as one-boson-exchange (OBE) models [4], the chiral perturbation theory [5], chiral quark models [6], and so on. In 2015, the LHCb Collaboration announced the observation of the hidden charm pentaquarks $P_{c}(4380)$ and $P_{c}(4450)$ [7-9]. The discovery reignited the interest of theorists and experimentalists in the pentaquark states [10]. There are many theoretical analyses devoted to the hidden charm pentaquarks [11-16].

Inspired by the hidden charm pentaquark states, people are also interested in the hidden strange pentaquark states, which are composed of $q q q s \bar{s}$. In fact, as early as 1999 , the evidence for a new baryon state with hidden strange and a mass of $>1.8 \mathrm{GeV}$ was reported by the SPHINX Collaboration in studying hyperon-kaon mass spectra in several proton diffractive reactions [17]. In 2001, the $N \phi$ bound state was proposed by Gao et al. [18], following the idea of Brodsky [19]. The calculation of the $N \phi$ system in Ref. [18] shows that the QCD

\footnotetext{
*hxhuang@njnu.edu.cn

†jlping@njnu.edu.cn
}

Published by the American Physical Society under the terms of the Creative Commons Attribution 4.0 International license. Further distribution of this work must maintain attribution to the author(s) and the published article's title, journal citation, and DOI. Funded by $S C O A P^{3}$.
Van der Waals attractive force [20], mediated by multigluon exchanges, can be strong enough to form the $N \phi$ bound state. It is also pointed out that the subthreshold quasifree $\phi$ meson photoproduction inside a nuclear medium will enhance the probability of the formation of the $N \phi$ bound state [18]. Therefore, further theoretical and experimental investigations are needed to understand dynamics of the pentaquark states with hidden strange.

To search for the $N \phi$ state experimentally, many groups carry out the study of $\phi$ meson production in nuclei, for example, the KEK-PS-E325 Collaboration [21], the LEPS Collaboration at Spring8/Osaka [22], the CLAS Collaboration at the Jefferson Lab [23], and the Jülich group [24]. The hidden strange molecular states are also supported by analyses of experimental data of the relevant photoproductions. In Ref. [25], it is pointed out that the $\phi$ meson should form bound states with all the nuclei considered by solving the Klein-Gordon equation with complex optical potentials. In Ref. [26,27], the $\phi$ meson properties in cold nuclear matter are investigated by implementing resonant $N \phi$ interactions. Recently, the Belle Collaboration reported their search for the decay of $\Lambda_{c}^{+} \rightarrow \pi^{0} p \phi$ in which no significant signal was observed with an upper limit on the branching fraction of $B\left(\Lambda_{c}^{+} \rightarrow \pi^{0} p \phi\right)<15.3 \times 10^{-5}$ at a $90 \%$ confidence level [28].

According to the experimental information, in the vicinity of $2 \mathrm{GeV}$, a $N \phi$ bound state is predicted in several models $[18,29-33]$. Xie and Guo studied the possible $\phi p$ resonance in the $\Lambda_{c}^{+} \rightarrow \pi^{0} p \phi$ decay by considering a triangle singularity mechanism on the basis of the investigation of the hidden strange pentaquark [29]. In Ref. [30], the $N \phi$ resonance state was obtained by channel coupling in the quark delocalization color screening model (QDCSM). They also performed a Monte Carlo simulation of the bound state production with an electron beam and a gold target, and found it was feasible to experimentally search for the $N \phi$ bound state through the near-threshold $\phi$ meson production from heavy nuclei. In Ref. [31] the authors showed that the $N \phi$ state is a quasibound state by considering channelcoupling of $N \phi$ and $\Lambda K^{*}$ in the chiral quark model (ChQM). In Ref. [32] it was shown that a bound state can be produced from the $N \phi$ interaction with spin-parity $3 / 2^{-}$after 
introduction of a Van der Waals force between the nucleon and $\phi$ meson. Moreover, the lattice QCD calculation also supports the existence of such kind of bound state [33]. Hence, it is worthwhile to make a systematical study of $N \phi$ bound states using the different methods in both experiment and theory, which will deepen our understanding about the hidden strange pentaquarks.

It is obvious that there is no common flavor quark between $N$ and $\phi$, which is similar to the dibaryon state $N \Omega$. In Ref. [34], we studied the strange dibaryon $N \Omega$ in both QDCSM and ChQM, and similar results were obtained. This indicates the consistency of describing $N \Omega$ in these two quark models. It is interesting to investigate whether or not coincident results of the $N \phi$ state can be obtained in both models. As we mentioned above, we have studied the $N \phi$ state in QDCSM. So, in the present work, we will study the $N \phi$ state in ChQM and the effect of channel coupling will also be investigated. This work will be helpful to understand the nucleon- $\phi$ meson interaction and the coupled-channel effect.

This paper is organized as follows. First, the chiral quark model is introduced briefly. The results for the $N \phi$ state are shown in Sec. III, with some discussions. Finally, the summary is given in Sec. IV.

\section{THE CHIRAL QUARK MODEL}

The chiral quark model has resulted in great achievements in describing hadron spectra, nucleon-nucleon interactions, and multiquark states [6]. In this model, the massive constituent quarks interact with each other through Goldstone boson exchange in addition to one-gluon exchange. Color confinement and scalar octet meson exchange are also introduced. The Hamiltonian of ChQM for the present calculation takes the form

$$
\begin{aligned}
& H=\sum_{i=1}^{5}\left(m_{i}+\frac{p_{i}^{2}}{2 m_{i}}\right)-T_{C M}+\sum_{j>i=1}^{5}\left(V_{i j}^{C}+V_{i j}^{G}+V_{i j}^{B}+V_{i j}^{\sigma}\right), \\
& V_{i j}^{C}=-a_{c} \lambda_{i}^{c} \cdot \lambda_{j}^{c}\left(r_{i j}^{2}+v_{0}\right), \\
& V_{i j}^{G}=\frac{1}{4} \alpha_{s} \lambda_{i}^{c} \cdot \lambda_{j}^{c}\left[\frac{1}{r_{i j}}-\frac{\pi}{2} \delta\left(\boldsymbol{r}_{i j}\right)\left(\frac{1}{m_{i}^{2}}+\frac{1}{m_{j}^{2}}+\frac{4 \boldsymbol{\sigma}_{i} \cdot \boldsymbol{\sigma}_{j}}{3 m_{i} m_{j}}\right)-\frac{3}{4 m_{i} m_{j} r_{i j}^{3}} S_{i j}\right] \\
& V_{i j}^{B}=V_{\pi}\left(\boldsymbol{r}_{i j}\right) \sum_{a=1}^{3} \lambda_{i}^{a} \cdot \lambda_{j}^{a}+V_{K}\left(\boldsymbol{r}_{i j}\right) \sum_{a=4}^{7} \lambda_{i}^{a} \cdot \lambda_{j}^{a}+V_{\eta}\left(\boldsymbol{r}_{i j}\right)\left[\left(\lambda_{i}^{8} \cdot \lambda_{j}^{8}\right) \cos \theta_{P}-\left(\lambda_{i}^{0} \cdot \lambda_{j}^{0}\right) \sin \theta_{P}\right] \\
& V_{\chi}\left(\boldsymbol{r}_{i j}\right)=\frac{g_{c h}^{2}}{4 \pi} \frac{m_{\chi}^{2}}{12 m_{i} m_{j}} \frac{\Lambda_{\chi}^{2}}{\Lambda_{\chi}^{2}-m_{\chi}^{2}} m_{\chi}\left\{\left(\boldsymbol{\sigma}_{i} \cdot \boldsymbol{\sigma}_{j}\right)\left[Y\left(m_{\chi} r_{i j}\right)-\frac{\Lambda_{\chi}^{3}}{m_{\chi}^{3}} Y\left(\Lambda_{\chi} r_{i j}\right)\right]\right. \\
& \left.+\left[H\left(m_{\chi} r_{i j}\right)-\frac{\Lambda_{\chi}^{3}}{m_{\chi}^{3}} H\left(\Lambda_{\chi} r_{i j}\right)\right] S_{i j}\right\}, \quad \chi=\pi, K, \eta \text {, } \\
& V_{i j}^{\sigma}=-\frac{g_{c h}^{2}}{4 \pi} \frac{\Lambda_{\sigma}^{2}}{\Lambda_{\sigma}^{2}-m_{\sigma}^{2}} m_{\sigma}\left[Y\left(m_{\sigma} r_{i j}\right)-\frac{\Lambda_{\sigma}}{m_{\sigma}} Y\left(\Lambda_{\sigma} r_{i j}\right)\right] \text {, } \\
& S_{i j}=\left\{3 \frac{\left(\boldsymbol{\sigma}_{i} \cdot \boldsymbol{r}_{i j}\right)\left(\boldsymbol{\sigma}_{j} \cdot \boldsymbol{r}_{i j}\right)}{r_{i j}^{2}}-\boldsymbol{\sigma}_{i} \cdot \boldsymbol{\sigma}_{j}\right\}, \\
& H(x)=\left(1+3 / x+3 / x^{2}\right) Y(x), \quad Y(x)=e^{-x} / x \text {. }
\end{aligned}
$$

where $T_{C M}$ is the center-of-mass kinetic energy and $\sigma, \lambda^{c}, \lambda^{a}$ are the SU(2) Pauli, SU(3) color, SU(3) flavor Gell-Mann matrices, respectively. The subscripts $i, j$ denote the quark index in the system. $Y(x)$ and $H(x)$ are the standard Yukawa functions [6], $\Lambda_{\chi}$ is the chiral symmetry breaking scale, and $\alpha_{s}$ is the effective scale-dependent running quark-gluon coupling constant [35],

$$
\alpha_{s}(\mu)=\frac{\alpha_{0}}{\ln \left(\frac{\mu^{2}+\mu_{0}^{2}}{\Lambda_{0}^{2}}\right)},
$$

where $\mu$ is the reduced mass of the interacting quarks pair. $\frac{g_{c h}^{2}}{4 \pi}$ is the chiral coupling constant for scalar and pseudoscalar chiral field coupling, determined from the $\pi$-nucleon-nucleon coupling constant through

$$
\frac{g_{c h}^{2}}{4 \pi}=\left(\frac{3}{5}\right)^{2} \frac{g_{\pi N N}^{2}}{4 \pi} \frac{m_{u, d}^{2}}{m_{N}^{2}} .
$$

To study the $u, d, s$ three-flavor states, the chiral SU(2) quark model has to be extended to the chiral SU(3) quark model [36,37]. There are two ways to introduce the scalar meson exchange. One is to use only $\sigma$ meson exchange between any quark pair; another is to introduce the full SU(3) scalar nonet meson exchange. In this work, both ways are employed in the dynamical study of $N \phi$ interaction in the framework of the resonating group method (RGM) [38]. To 
TABLE I. The parameters of the models: $m_{\pi}=0.7 \mathrm{fm}^{-1}, m_{k}=$ $2.51 \mathrm{fm}^{-1}, m_{\eta}=2.77 \mathrm{fm}^{-1}, m_{\sigma}=3.42 \mathrm{fm}^{-1}, m_{a_{0}}=m_{\kappa}=m_{f_{0}}=$ $4.97 \mathrm{fm}^{-1}, \Lambda_{\pi}=4.2 \mathrm{fm}^{-1}, \Lambda_{K}=5.2 \mathrm{fm}^{-1}, \Lambda_{\eta}=5.2 \mathrm{fm}^{-1}, \Lambda_{\sigma}=$ $4.2 \mathrm{fm}^{-1}, \quad \Lambda_{a_{0}}=\Lambda_{\kappa}=\Lambda_{f_{0}}=5.2 \mathrm{fm}^{-1}, g_{c h}^{2} /(4 \pi)=0.54, \quad \theta_{p}=$ $-15^{0}$.

\begin{tabular}{lccc}
\hline \hline & ChQM1 & ChQM2 & ChQM3 \\
\hline$b(\mathrm{fm})$ & 0.518 & 0.518 & 0.518 \\
$m_{u}(\mathrm{MeV})$ & 313 & 313 & 313 \\
$m_{d}(\mathrm{MeV})$ & 313 & 313 & 313 \\
$m_{s}(\mathrm{MeV})$ & 573 & 536 & 573 \\
$a_{c}(\mathrm{MeV})$ & 48.59 & 48.59 & 48.59 \\
$v_{0}(\mathrm{MeV})$ & -1.2145 & -1.2145 & -0.961 \\
$\alpha_{0}$ & 0.510 & 0.510 & 0.583 \\
$\left.\Lambda_{0}(\mathrm{fm})^{-1}\right)$ & 1.525 & 1.525 & 1.616 \\
$\mu_{0}(\mathrm{MeV})$ & 445.808 & 445.808 & 422.430 \\
\hline \hline
\end{tabular}

check the model dependence of the results, we use three kinds of chiral quark models: (1) ChQM1, $\sigma$ meson exchange is used between any quark pair; (2) ChQM2, $\sigma$ meson exchange is only valid for a $u$ and/or $d$ quark pair; (3) ChQM3, the full SU(3) scalar nonet-meson exchange is employed. These scalar nonet-meson exchange potentials [36] have the same form as the $\sigma$ meson exchange of the $\mathrm{SU}(2) \mathrm{ChQM}$, that is,

$$
\begin{aligned}
V_{i j}^{\sigma_{a}}= & V_{a_{0}}\left(\boldsymbol{r}_{i j}\right) \sum_{a=1}^{3} \lambda_{i}^{a} \cdot \lambda_{j}^{a}+V_{\kappa}\left(\boldsymbol{r}_{i j}\right) \sum_{a=4}^{7} \lambda_{i}^{a} \cdot \lambda_{j}^{a} \\
& +V_{f_{0}}\left(\boldsymbol{r}_{i j}\right) \lambda_{i}^{8} \cdot \lambda_{j}^{8}+V_{\sigma}\left(\boldsymbol{r}_{i j}\right) \lambda_{i}^{0} \cdot \lambda_{j}^{0}, \\
V_{k}\left(\boldsymbol{r}_{i j}\right)= & -\frac{g_{c h}^{2}}{4 \pi} \frac{\Lambda_{k}^{2} m_{k}}{\Lambda_{k}^{2}-m_{k}^{2}}\left[Y\left(m_{k} r_{i j}\right)-\frac{\Lambda_{k}}{m_{k}} Y\left(\Lambda_{k} r_{i j}\right)\right],
\end{aligned}
$$

with $k=a_{0}, \kappa, f_{0}$, and $\sigma$.

All the other symbols in the above expressions have their usual meanings, and all the parameters needed in the present calculation are taken from Ref. [34] and listed in Table I. The calculated baryon and meson masses are presented in Table II with the experimental values. From Table II, we can see that the baryon masses are fitted well while the meson masses are not. This is a deficiency of the quark model: baryons and mesons cannot be described well with the same set of model parameters. In discussing the energy of pentaquark system, subtraction procedures are adopted to reduce the effect of the mismatching of meson masses, which will be explained in Sec. III.

TABLE II. The masses of ground-state baryons and mesons (in $\mathrm{MeV})$.

\begin{tabular}{lccccccc}
\hline \hline & $N$ & $\Lambda$ & $\Sigma$ & $\Sigma^{*}$ & $\phi$ & $K$ & $K^{*}$ \\
\hline ChQM1 & 939 & 1124 & 1239 & 1360 & 1056 & 695 & 817 \\
ChQM2 & 939 & 1137 & 1245 & 1376 & 1016 & 686 & 817 \\
ChQM3 & 939 & 1123 & 1267 & 1344 & 1054 & 667 & 840 \\
Expt. & 939 & 1116 & 1193 & 1385 & 1019 & 498 & 892 \\
\hline \hline
\end{tabular}

TABLE III. The symmetries of colorful $q q q$ and $q \bar{q}$ clusters.

\begin{tabular}{lccccccc}
\hline \hline & $\Delta^{\prime}$ & $\Sigma^{* \prime}$ & $\Xi^{* \prime}$ & $N^{\prime \prime}$ & $\Lambda^{\prime \prime}$ & $\Sigma^{\prime \prime}$ & $\Lambda_{s}^{\prime}$ \\
\hline$[c]$ & {$[21]$} & {$[21]$} & {$[21]$} & {$[21]$} & {$[21]$} & {$[21]$} & {$[21]$} \\
{$[\sigma]$} & {$[21]$} & {$[21]$} & {$[21]$} & {$[3]$} & {$[3]$} & {$[3]$} & {$[21]$} \\
{$[f]$} & {$[3]$} & {$[3]$} & {$[3]$} & {$[21]$} & {$[21]$} & {$[21]$} & {$[111]$} \\
$I$ & $\frac{3}{2}$ & 1 & $\frac{1}{2}$ & $\frac{1}{2}$ & 0 & 1 & 0 \\
$S$ & 0 & 1 & 2 & 0 & 1 & 1 & 1 \\
& $\eta^{\prime}$ & $\phi^{\prime}$ & $K^{* \prime}$ & $K_{s}^{* \prime}$ & & & \\
\hline$[c]$ & {$[21]$} & {$[21]$} & {$[21]$} & {$[21]$} & & & \\
{$[\sigma]$} & {$[11]$} & {$[2]$} & {$[11]$} & {$[2]$} & & & \\
{$[f]$} & {$[21]$} & {$[21]$} & {$[21]$} & {$[21]$} & & & \\
$I$ & 0 & 0 & $\frac{1}{2}$ & $\frac{1}{2}$ & & & \\
$S$ & 2 & 2 & 1 & 1 & & & \\
\hline \hline
\end{tabular}

\section{RESULTS AND DISCUSSION}

Here, we investigate the properties of the $N \phi$ state with quantum numbers of $I J^{P}=\frac{1}{2} \frac{3}{2}^{-}$within the chiral quark models mentioned above. The main goal of this work is to investigate whether the $N \phi$ state is bound or not in ChQM. A dynamic calculation based on RGM, a well established method for studying the bound or scattering five-quark states, was done here. Expanding the relative motion wave function between two clusters by employing well-defined basis wave functions, such as Gaussian functions, the integrodifferential equation of RGM transforms into an algebraic equation, a generalized eigenequation. Then one can solve the generalized eigenequation for a bound-state problem and obtain the corresponding binding energy. The details of solving the RGM equation can be found in Refs. [39-41].

In our calculation, we consider the effect of channel coupling including both the color-singlet channels and the hidden-color channels. Color singlet means the color symmetries of the $q q q$ cluster and $q \bar{q}$ cluster are all [111], and hidden color indicates that the color symmetries of them are all [21]. The colorful $q q q$ cluster and $q \bar{q}$ cluster are listed in Table III. The color symmetry $[c]$, spin symmetry $[\sigma]$, flavor symmetry $[f]$, isospin $I$, and strangeness $S$ of the $q q q$ and $q \bar{q}$ clusters are also listed in the table. Moreover, the labels of all 16 channels of the $N \phi$ system are listed in Table IV. The first five channels are color-singlet ones, others are hidden-color ones. The baryon-meson separation is taken to be less than 6 $\mathrm{fm}$ for the bound-state calculation of this system.

Since an attractive potential is necessary for forming bound state or resonance, the effective potentials between $N$ and $\phi$ in the three quark models are calculated and shown in Fig. 1. The effective potential between two colorless clusters is defined as $V(s)=E(s)-E(\infty)$, where $E(s)$ is the diagonal matrix

TABLE IV. Channels of the $N \phi$ system.

\begin{tabular}{lccccccc}
\hline \hline 1 & 2 & 3 & 4 & 5 & 6 & 7 & 8 \\
$N \phi$ & $\Lambda K^{*}$ & $\Sigma K^{*}$ & $\Sigma^{*} K$ & $\Sigma^{*} K^{*}$ & $\Delta^{\prime} \phi^{\prime}$ & $\Sigma^{* \prime} K^{* \prime}$ & $\Xi^{* \prime} K^{* \prime}$ \\
\hline 9 & 10 & 11 & 12 & 13 & 14 & 15 & 16 \\
$N^{\prime \prime} \eta^{\prime}$ & $N^{\prime \prime} \phi^{\prime}$ & $\Lambda^{\prime \prime} K^{\prime}$ & $\Lambda^{\prime \prime} K^{* \prime}$ & $\Sigma^{\prime \prime} K^{\prime}$ & $\Sigma^{\prime \prime} K^{* \prime}$ & $\Sigma^{* \prime} K^{* \prime}$ & $\Lambda_{s}^{\prime} K^{* \prime}$ \\
\hline \hline
\end{tabular}



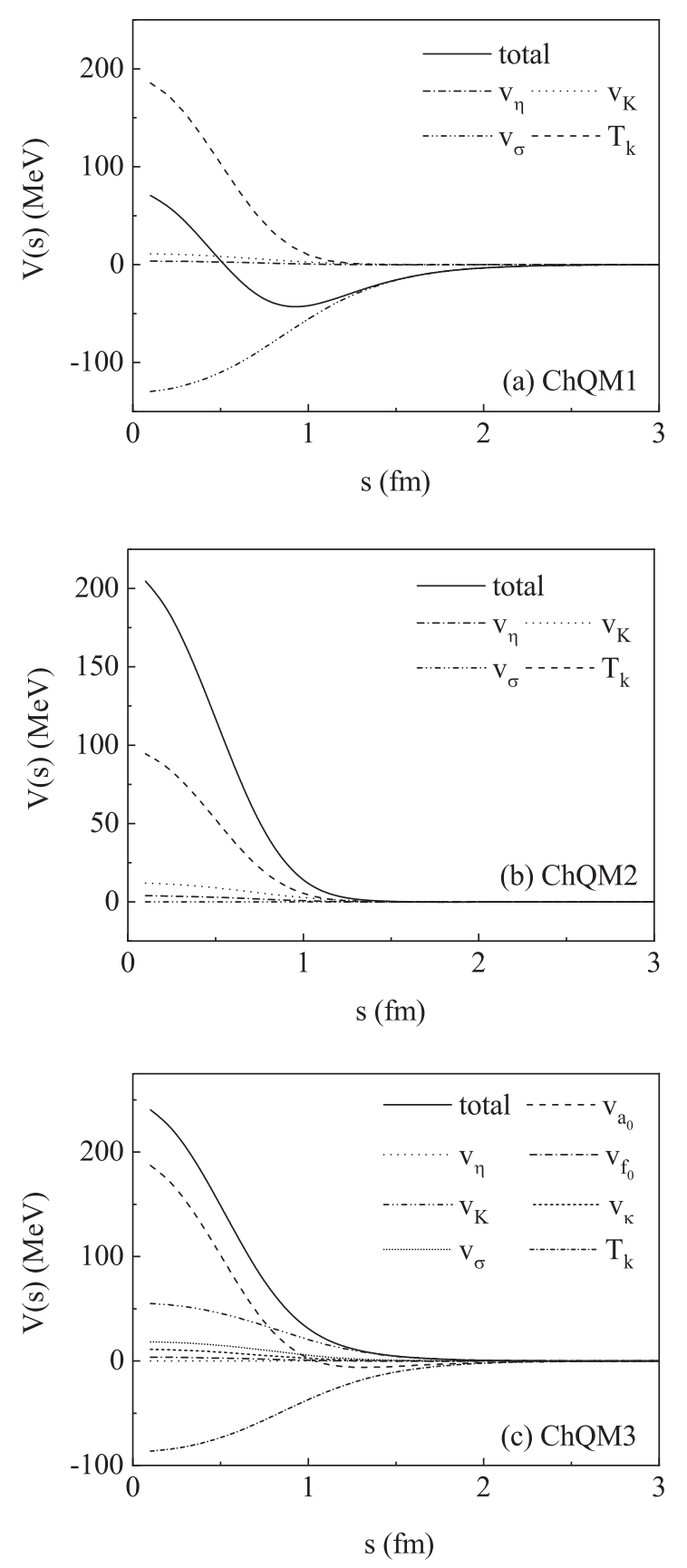

FIG. 1. The contributions of various terms of the Hamiltonian to the effective potentials.

element of the Hamiltonian of the system in the generating coordinate. The subtraction is also to abate the effect of the mismatching of meson masses. One sees that the potentials of the $N \phi$ state in both ChQM1 and ChQM3 are attractive, while the one in ChQM2 is repulsive. Besides, the attraction in ChQM1 is much larger than the one in ChQM3.

To investigate the contribution of each interaction term to the total effective potentials between $N$ and $\phi$, we also calculate the contribution from the kinetic energy $\left(T_{k}\right)$, the one-boson exchange $\left(V_{\pi}, V_{k}\right.$, and $\left.V_{\eta}\right)$, and the scalar octetmeson exchange $\left(V_{a_{0}}, V_{f_{0}}, V_{\kappa}, V_{\sigma}\right)$. Here, the interactions of
TABLE V. The binding energies B with channel coupling.

\begin{tabular}{lccc}
\hline \hline & $B_{s c}(\mathrm{MeV})$ & $B_{5 c c}(\mathrm{MeV})$ & $B_{16 c c}(\mathrm{MeV})$ \\
\hline ChQM1 & $\mathrm{ub}$ & -5.70 & -12.27 \\
ChQM2 & $\mathrm{ub}$ & $\mathrm{ub}$ & $\mathrm{ub}$ \\
ChQM3 & $\mathrm{ub}$ & $\mathrm{ub}$ & $\mathrm{ub}$ \\
\hline \hline
\end{tabular}

the one-gluon exchange $\left(V^{G}\right)$, the confinement $\left(V^{C}\right)$ term, the one-pion exchange $\left(V_{\pi}\right)$, and the $a_{0}$-meson exchange $\left(V_{a_{0}}\right)$ do not work between $N$ and $\phi$, because there are no common flavor quark between these two clusters. The contributions of other terms to the effective potential are show in Figs. 1(a)1(c). In ChQM1, the potentials of the $\eta, K$, and the kinetic energy $\left(T_{k}\right)$ are all repulsive and the $\sigma$ meson exchange interaction is attractive. The total potential is attractive. This means it is possible to form a $N \phi$ bound state in ChQM1. In ChQM2, where the $\sigma$ meson is restricted to exchange between a $u$ and $d$ quark pair only, this indicates that the $\sigma$ meson exchange potential would have no contribution to the interaction between $N$ and $\phi$. It can be found from Fig. 1(b) that the total potential is repulsive. In ChQM3, as can be seen in Fig. 1(c), although the $\sigma$ meson exchange potential yields a great deal of attraction, the potentials of $\eta, K, f_{0}, \kappa$ and the kinetic energy are all repulsive. The repulsion is sufficient to counteract the attraction of $\sigma$ meson exchange, which causes very weak attraction of the total potential for the $N \phi$ state. All these properties are consistent with our previous study of the $N \Omega$ state [34], where there is no common flavor quark between $N$ and $\Omega$.

In order to see whether or not there is any bound state, we continue to do a dynamic calculation. The binding energies of the $N \phi$ state are listed in Table $\mathrm{V}$, where $B_{s c}$ means the binding energy of the single-channel calculation of the $N \phi$ state; $B_{5 c c}$ indicates that the binding energy is produced by the coupling of five color-singlet channels; and $B_{16 c c}$ stands for the binding energy of all 16 channels coupling. From Table V one can see that the single channel $N \phi$ is unbound (labeled as "ub" in Table V) in all three quark models. This means that the attraction between $N$ and $\phi$ in ChQM1 is not large enough to from a bound state; also the attraction between $N$ and $\phi$ in ChQM3 is too weak to make the $N \phi$ bound. This is also reasonable for the unbound $N \phi$ state in ChQM2 because of the repulsive interaction between $N$ and $\phi$.

Next we take into account the effects of channel coupling. From Table V, we can see that in ChQM1 the $N \phi$ state can be bound by the coupling of the color-singlet channels. Moreover, the binding energy can be larger by the coupling of the hidden-color channels. This means that channel coupling has an important impact on the $N \phi$ state. These results are consistent with our previous work on the $N \phi$ state in QDCSM [30], in which the $N \phi$ state was bound by channel coupling. However, the effect of channel coupling is still not enough to generate the binding energy of the $N \phi$ state in both ChQM2 and ChQM3. In ChQM3, although $\sigma$-meson exchange plays the same role as in ChQM1, the other scalar mesons in the nonet play the role of repulsion, which leads to too weak attraction between $N$ and $\phi$. In QDCSM, the color screening 


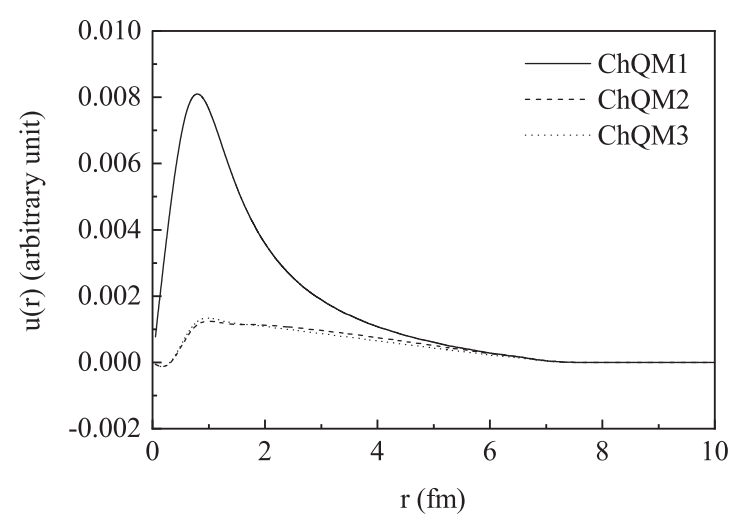

FIG. 2. The relative motion wave function of the $N \phi$ state in three quark models.

and quark delocalization contribute the attraction between two clusters, which are less flavor dependent. So multiquark resonances with strangeness are good places to distinguish the intermediate-range attraction between two clusters.

Moreover, we can obtain the relative motion wave function $u(R)$ for the $N \phi$ state, which is expanded by Gaussian functions with different centers. The relative motion wave function of the $N \phi$ state in three models is shown in Fig. 2. The shapes of lines indicate that the $N \phi$ state in ChQM1 is bound, while it is unbound in ChQM2 and ChQM3.

Finally, we should mention how we obtain the mass of a hidden strange molecular pentaquark in this work. Generally, the mass of a molecular pentaquark can be written as $M^{t h}=M_{1}^{t h}+M_{2}^{t h}+B$, where $M_{1}^{t h}$ and $M_{2}^{t h}$ stand for the theoretical masses of a baryon and a meson, respectively, and $B$ is the binding energy of the molecular states. In order to minimize the theoretical errors and to compare calculated results to the experimental data, we shift the mass of the molecular pentaquark to $M=M_{1}^{\text {exp }}+M_{2}^{\text {exp }}+B$, where the experimental values of the baryon and the meson are used. This is the subtraction procedure mentioned in Sec. II. By using this subtraction procedure, we can obtain the mass of the $N \phi$ state in ChQM1 by 16-channel coupling; it is $M=$ $939+1020+(-12.27)=1946.73(\mathrm{MeV})$.

\section{SUMMARY}

In this paper, we look for the $N \phi$ bound state within three chiral quark models: ChQM1, ChQM2, and ChQM3, which refer to the different kinds of $\sigma$ meson exchange. The calculated results show the following: (1) The potentials of the $N \phi$ state in both ChQM1 and ChQM3 are attractive, while the one in ChQM2 is repulsive. The attraction in ChQM1 is much stronger than that in ChQM3. (2) Although the single $N \phi$ is unbound in ChQM1, a bound $N \phi$ state can be obtained by the effect of channel coupling. This means that the channel coupling effect in this model has an important influence on the existence of the bound state. (3) The $N \phi$ is obviously unbound in ChQM2 because of the repulsive interaction between $N$ and $\phi$. (4) In ChQM3, the attraction between $N$ and $\phi$ is too weak to make the $N \phi$ bound.

The hidden charm pentaquark candidates have been reported by $\mathrm{LHCb}$; we also expect the existence of the hidden strange pentaquark $N \phi$ state. The theoretical study of pentaquark systems can help us to understand the nature of multiquark states. The experimental search for pentaquark systems will not only test the quark model, but also help us to understand the fundamental strong interaction theory, QCD. In the near future, more and more novel phenomena are expected with experimental progress, especially from $\mathrm{LHCb}$ and Belle II. Clearly, the subject deserves effort using both theory and experiment.

\section{ACKNOWLEDGMENTS}

This work is supported partly by the National Natural Science Foundation of China under Contracts No. 11675080, No. 11775118 , and No. 11535005 , and by the Natural Science Foundation of the Jiangsu Higher Education Institutions of China (Grant No. 16KJB140006).
[1] N. Ishii, S. Aoki, and T. Hatsuda, Phys. Rev. Lett. 99, 022001 (2007).

[2] C. Alexandrou, P. de Forcrand, and A. Tsapalis, Phys. Rev. D 65, 054503 (2002); T. T. Takahashi, H. Suganuma, Y. Nemoto, and H. Matsufuru, ibid. 65, 114509 (2002).

[3] F. Okiharu, H. Suganuma, and T. T. Takahashi, Phys. Rev. Lett. 94, 192001 (2005).

[4] R. Machleidt, The Meson Theory of Nuclear Forces and Nuclear Structure, edited by J. W. Negele and E. Vogt, Advances in Nuclear Physics (Springer, Boston, MA, 1989), Vol. 19, pp. 189-376.

[5] E. Epelbaum, H. W. Hammer, and Ulf-G. Meissner, Rev. Mod. Phys. 81, 1773 (2009) and references therein.

[6] A. Valcarce, H. Garcilazo, F. Fernández, and P. Gonzalez, Rep. Prog. Phys. 68, 965 (2005) and references therein.

[7] R. Aaij et al. (LHCb Collaboration), Phys. Rev. Lett. 115, 072001 (2015).
[8] R. Aaij et al. (LHCb Collaboration), Phys. Rev. Lett. 117, 082002 (2016).

[9] R. Aaij et al. (LHCb Collaboration), Phys. Rev. Lett. 117, 082003 (2016).

[10] H. X. Chen, W. Chen, X. Liu, and S. L. Zhu, Phys. Rep. 639, 1 (2016).

[11] R. Chen, X. Liu, X. Q. Li, and S. L. Zhu, Phys. Rev. Lett. 115, 132002 (2015).

[12] J. He, Phys. Lett. B 753, 547 (2016).

[13] H. X. Chen, W. Chen, X. Liu, T. G. Steele, and S. L. Zhu, Phys. Rev. Lett. 115, 172001 (2015).

[14] M. Karliner and J. L. Rosner, Phys. Rev. Lett. 115, 122001 (2015).

[15] H. X. Huang, C. R. Deng, J. L. Ping, and F. Wang, Eur. Phys. J. C 76, 624 (2016).

[16] Y. Shimizu, D. Suenaga, and M. Harada, Phys. Rev. D 93, 114003 (2016). 
[17] S. V. Golovkin et al. (SPHINX Collaboration), Eur. Phys. J. A 5, 409 (1999).

[18] H. Gao, T.-S. H. Lee, and V. Marinov, Phys. Rev. C 63, 022201(R) (2001).

[19] S. J. Brodsky, I. Schmidt, and G. F. de Teramond, Phys. Rev. Lett. 64, 1011 (1990).

[20] T. Appelquist and W. Fischler, Phys. Lett. B 77, 405 (1978).

[21] R. Muto, J. Chiba, H. Enyo et al. (KEK-PS E325 Collaboration), Phys. Rev. Lett. 98, 042501 (2007).

[22] T. Ishikawa, D. S. Ahn, J. K. Ahn, H. Akimune, W. C. Chang, S. Date, H. Fujimura and M. Fujiwara et al., Phys. Lett. B 608, 215 (2005).

[23] M. H. Wood et al. (CLAS Collaboration), Phys. Rev. Lett. 105, 112301 (2010).

[24] M. Hartmann, Y. T. Kiselev, A. Polyanskiy, E. Y. Paryev, M. Buscher, D. Chiladze, S. Dymov, A. Dzyuba et al., Phys. Rev. C 85, 035206 (2012).

[25] J. J. Cobos-Martinez, K. Tsushima, G. Krein, and A. W. Thomas, Phys. Rev. C 96, 035201 (2017).

[26] D. Cabrera, A. N. Hiller Blin, and M. J. Vicente Vacas, Phys. Rev. C 95, 015201 (2017).

[27] D. Cabrera, A. N. Hiller Blin, M. J. Vicente Vacas, and P. Fernández de Córdoba, Phys. Rev. C 96, 034618 (2017).

[28] B. Pal et al. (Belle Collaboration), Phys. Rev. D 96, 051102 (2017)
[29] J. J. Xie and F. K. Guo, Phys. Lett. B 774, 108 (2017).

[30] H. Gao, H. Huang, T. Liu, J. Ping, F. Wang, and Z. Zhao, Phys. Rev. C 95, 055202 (2017).

[31] F. Huang, Z.-Y. Zhang, and Y.-W. Yu, Phys. Rev. C 73, 025207 (2006)

[32] J. He, H. Huang, D. Y. Chen, and X. Zhu, arXiv:1804.09383.

[33] S. R. Beane, E. Chang, S. D. Cohen, W. Detmold, H.-W. Lin, K. Orginos, A. Parreno, and M. J. Savage, Phys. Rev. D 91, 114503 (2015)

[34] H. X. Huang, J. L. Ping, and F. Wang, Phys. Rev. C 92, 065202 (2015).

[35] J. Vijande, F. Fernandez, and A. Valcarce, J. Phys. G 31, 481 (2005).

[36] H. Garcilazo, T. Fernandez-Carames, and A. Valcarce, Phys. Rev. C 75, 034002 (2007).

[37] Q. B. Li, P. N. Shen, Z. Y. Zhang, and Y. W. Yu, Nucl. Phys. A 683, 487 (2001).

[38] A. J. Buchmann, Y. Yamauchi, and A. Faessler, Nucl. Phys. A 496, 621 (1989).

[39] K. Wildermuth and Y. C. Tang, A Unified Theory of the Nucleus (Vieweg, Braunschweig, 1977).

[40] M. Kamimura, Suppl. Prog. Theor. Phys. 62, 236 (1977).

[41] M. Oka and K. Yazaki, Prog. Theor. Phys. 66, 556 (1981). 